\title{
Phytobezoars, Small Bowel Obstruction, and Intestinal Infarction: The Case of the Grape Ileus
}

\author{
Anna Liveris, Michael Zeidman, and Peter K. Kim
}

\begin{abstract}
Background: The majority of small bowel obstructions (SBOs) that require operations are caused by adhesions after previous operations, hernias, and cancer. Undigested foods of the appropriate size are an unusual source of endoluminal SBOs.

Methods: Case report and review of the literature. An 83-year-old female with a history of dementia was admitted with increasing abdominal pain and tenderness. A computed tomography (CT) scan demonstrated a SBO with a small bowel mass at the transition point in the distal ileum. Urgent laparotomy revealed a closed loop SBO requiring an ileocectomy.

Results: Analysis of the specimen revealed an undigested grape and multiple raisins.

Conclusions: Phytobezoars are an unusual source of life-threatening SBO. Risk factors include poor dentition and dementia. The diagnosis can be made using careful history taking, physical exam, and appropriate imaging, preferably a CT scan. At risk patients should be counseled on appropriate dietary measures to prevent this dangerous condition.
\end{abstract}

A N 83-YEAR-OLD FEMALE with past medical history of diabetes mellitus, congestive heart failure, hypertension, hypothyroidism, and dementia was sent to the emergency department (ED) from her nursing home for severe acute onset of abdominal pain, nausea, and unrelenting non-bloody, non-bilious emesis. She admits constipation but denies fevers and chills. At the nursing home, she was taking multiple medications including beta-blockers, anti-psychotics, antihypertensives, a loop diuretic, and levothyroxine. Of note, she had no past surgical history.

\section{Physical Exam}

In the emergency department, she was afebrile at $99.1^{\circ} \mathrm{F}$ with a blood pressure of 132/69, and a heart rate of 121 beats per minute that was tachycardic. Her respiratory rate was 18 breaths per minute, and her oxygen saturation was $96 \%$ on $2 \mathrm{~L}$ nasal cannula. She appeared anxious, elderly, obese, and she was oriented only to person and place. She had one single tooth with poor dentition and was tachycardic with a normal s1 and s2. Her abdominal exam was significant for diffuse tenderness to light palpation with rebound and guarding, but no palpable masses or hernias.

Her labs were significant for an elevated WBC of 14.2 cells $/ \mathrm{nL}$ and lactate of $1.95 \mathrm{mmol} / \mathrm{L}$. All other labs including chemistries, liver function tests, and coagulation panel were within normal limits. Her electrocardiogram (EKG) showed a new left bundle branch block. A portable abdominal radiograph was done initially in the ED. It showed multiple distended and mildly dilated loops of small bowel likely due to a SBO (Fig. 1). Nasogastric tube placement was unsuccessful because of the patient's agitation and lack of cooperation. A CT scan of the abdomen and pelvis was done to evaluate for an obstructive mass given the lack of prior operations. The CT scan showed a soft tissue density observed within the lumen of the distal small bowel, interpreted by the radiologist as a small intraluminal lesion or intussusception (Figs. 2A and 2B).

\section{Intervention}

Given the patient's worsening condition during the abdominal exam, particularly concerning for peritonitis and

Department of Surgery, Jacobi Medical Center, Albert Einstein College of Medicine, Bronx, New York.

(C) Anna Liveris et al. 2016; Published by Mary Ann Liebert, Inc. This Open Access article is distributed under the terms of the Creative Commons License (http://creativecommons.org/licenses/by/4.0), which permits unrestricted use, distribution, and reproduction in any medium, provided the original work is properly credited. 


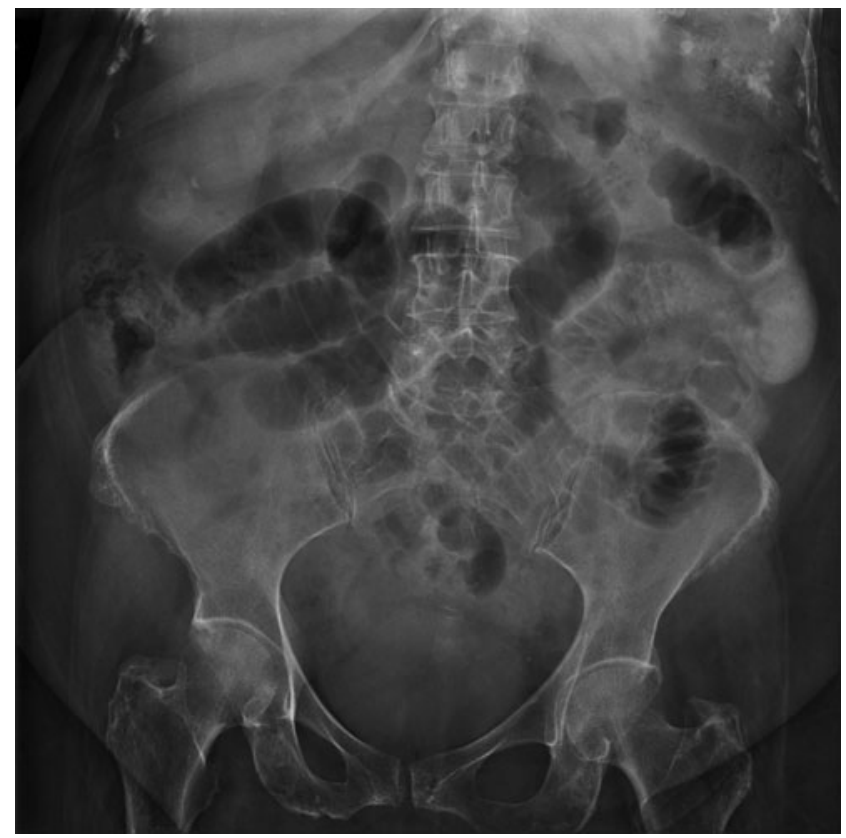

FIG. 1. Abdominal radiograph demonstrated dilated loops of small bowel consistent with intestinal obstruction in a patient with no previous surgical history.

possible obstructive mass without appropriate nasogastric decompression, the decision was made to perform an emergent exploratory laparotomy. Upon entry into the abdomen via a midline incision, a large amount of purulent fluid was suctioned. The small bowel was then examined from the ligament of Treitz to the terminal ileum where it appeared grossly thickened, and several adhesions were lysed. Upon close inspection, approximately $5 \mathrm{~cm}$ from the ileocecal (IC) valve, it appeared that a palpable endoluminal mass, approximately $2 \times 3 \mathrm{~cm}$ in size along with dense adhesions, had caused a volvulus of small bowel and a closed loop obstruction. The small bowel appeared gangrenous and nonviable, therefore an ileocectomy with side-to-side stapled anastomosis was performed. Subsequently, the small bowel was opened on the back table and it appeared that the bowel was filled with a $3-\mathrm{cm}$ intact grape as well as multiple raisins more proximally (Fig. 3). The abdomen was irrigated and closed primarily.

\section{Outcome}

The patient was transferred intubated to the surgical intensive care unit where cardiology monitored her for her pre-operative EKG findings and a worsening ejection fraction of $30 \%$ on post-operative EKG. Her medications were restarted after she was successfully extubated on postoperative day two. She remained stable hemodynamically and was transferred out of the intensive care unit. Postoperatively, her diet was advanced as her condition improved, and she was discharged back to her nursing home on post-operative day 12 .

\section{Discussion}

Small bowel obstruction is a substantial health and economic burden, accounting for more than 300,000 hospitaliza-
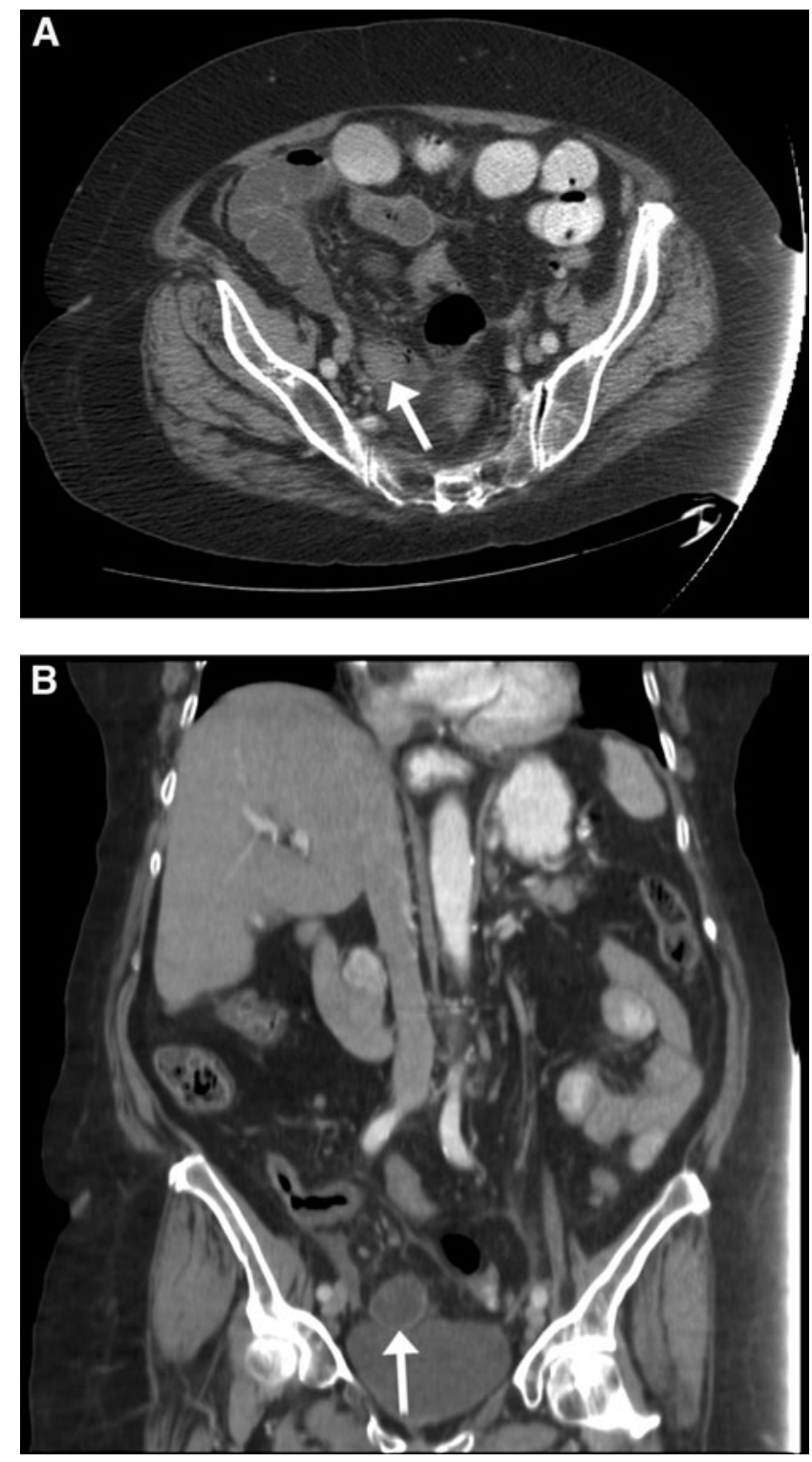

FIG. 2. (A) Axial view of CT scan of abdomen shows dilated small bowel and endoluminal mass (arrow) in the distal small bowel, and (B) coronal view of CT scan of the abdomen consistent with endoluminal mass (arrow) in the terminal ileum adjacent to the bladder.

tions, 30,000 deaths, and $\$ 3$ billion per year in the United States [1]. Significant advancements have been achieved from the days of Praxagoras, who described creating a diverting enterocutaneous fistula to relieve bowel obstruction in the fourth century B.C. However, SBO still remains a challenging entity in terms of diagnosis and timing of surgical intervention.

The etiologies of SBO can be classified broadly into either extra-luminal or intra-luminal causes. Extra-luminal sources are far more common, with post-operative adhesions alone accounting for the majority of all cases. Intra-luminal obstruction is usually caused by a primary tumor, although obstructing agents such as gallstones, foreign bodies, and bezoars have been reported. Phytobezoars, i.e., bezoars containing vegetable matter, are rare culprits that account for only $0.4-4 \%$ of all SBOs [2]. 


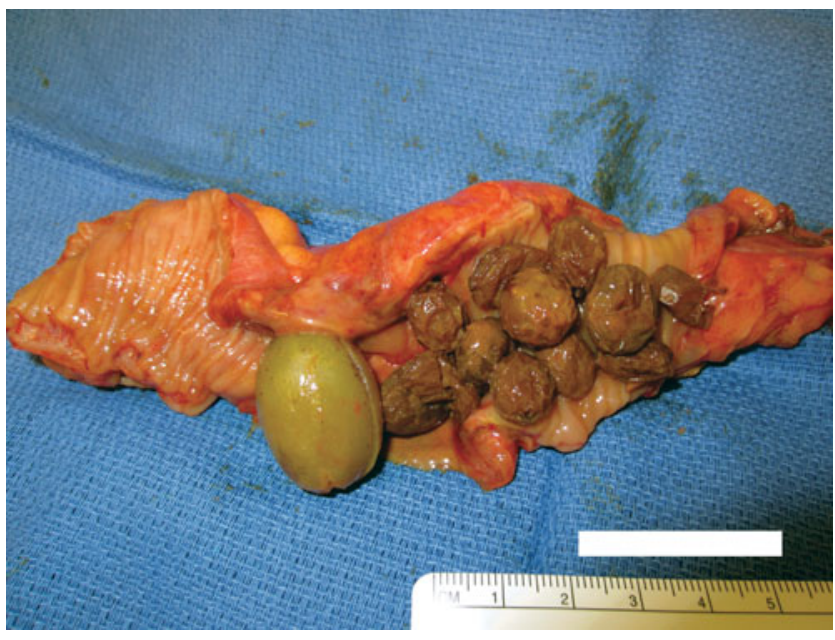

FIG. 3. The resected segment of ischemic small bowel was filled with raisins and a large undigested grape, phytobezoars that contributed to this patient's SBO.

Risk factors for phytobezoar-induced SBO include excessive ingestion of cellulose-containing foods, insufficient mastication or poor dental hygiene, and drugs or diseases that delay gastric or small bowel motility. Our patient was toothless, had poor oral hygiene, and had ingested many whole grapes and whole raisins a few days prior to admission. All of these factors contributed to her unusual presentation that required an operation.

There are only two previously reported cases of SBO caused by an intact grape $[3,4]$. The skin of a grape is composed mainly of water, cellulose, pectin, and tannins covered by a waxy layer, or cuticle, which gives the grape its waterproof properties. Dietary cellulose is mainly digested via fermentation by microflora in the colon, and remains relatively intact throughout the stomach and small bowel.

The diagnosis of SBO due to a phytobezoar is difficult to make on history and physical exam alone. Patients with phytobezoarinduced SBO present with the typical constellation of symptoms observed in SBO secondary to any cause: Abdominal pain, distension, nausea, vomiting, and obstipation. These symptoms may also be observed in patients with ileus, constipation, or large bowel obstruction. Therefore, imaging plays an important role, as these entities have different treatment modalities.

Radiological options used to identify phytobezoars include abdominal plain film (XR), small bowel follow through barium study, ultrasound (US), and CT. XR is typically the first option because of its low cost, speed, and minimal radiation. The outline of a phytobezoar may be identified, but this is often obscured by the air-fluid levels associated with SBO and difficult to distinguish between any other type of intraluminal mass [2]. A barium study would display a filling defect at the point of obstruction but has similar limitations as an XR. A phytobezoar, such as a peach pit, viewed under US might appear as a hyperechoic mass with prominent posterior acoustic shadowing, similar to the appearance of a calcified mass or gallstone, although not in this case. For hemodynamically instable patients, abdominal CT scan with oral contrast is the preferred imaging study for the diagnosis of SBO when a phytobezoar is suspected. CT not only provides the precise location of the phytobezoar but it may also distinguish a phytobezoar from other intra-luminal masses.
The treatment of SBO from a phytobezoar is an operation. Exploratory laparotomy is the procedure of choice with a meticulous examination of the small bowel, including the pylorus of the stomach. Some patients have multiple bezoars as was the case with our patient with the grape accompanied by several undigested raisins. The typical point of obstruction occurs in the distal ileum because of the natural tapering of the small bowel at this point. Our grape was found $5 \mathrm{~cm}$ proximal to the IC valve. An attempt may be made to express manually the bezoar past the IC valve; however, this maneuver was not indicated in our patient as she had a concomitant closed-loop obstruction with ischemia that necessitated an ileocecectomy.

Phytobezoars are a rare but life-threatening cause of SBO, as patients require an emergent operation and will not improve with conservative measures such as nasogastric tube decompression. The diagnosis can be made using careful history taking, physical exam, and appropriate imaging, preferably a CT scan. At-risk patients should be counseled on appropriate dietary measures to prevent this dangerous condition.

\section{Author Disclosure Statement}

Michael Zeidman, Anna Liveris, and Peter K. Kim report nothing to disclose.

\section{References}

1. Cappell M, Batke M. Mechanical obstruction of the small bowel and colon. Medic Clin of North America 2008;92:575-597.

2. Pujar KA, Sreekar PA, Hiremath B. Phytobezoar: A rare cause of small bowel obstruction. J Clin Diag Res 2013; 10:2298-2299.

3. Trotta M, Cesaretti M, Conzi R, et al. Elderly male with mesogastric pain. Ann Emerg Med 2011;58:e1-e2.

4. Cox J, Grigg M. Small bowel obstruction by an intact grape. J Amer Geriat Soc 1986;34:550.

Address correspondence to: Dr. Peter K. Kim Department of Surgery Albert Einstein College of Medicine Jacobi Medical Center 1400 Pelham Parkway Building 1, Room 510 Bronx, NY 10461

E-mail: Kim.peter.p01@gmail.com

$$
\begin{aligned}
& \text { Abbreviations Used } \\
\mathrm{CT} & =\text { computed tomography } \\
\mathrm{ED} & =\text { emergency department } \\
\mathrm{EKG} & =\text { electrocardiogram } \\
\mathrm{SBO} & =\text { small bowel obstruction } \\
\mathrm{US} & =\text { ultrasound } \\
\mathrm{XR} & =\text { plain film }
\end{aligned}
$$

Cite this article as: Liveris A, Zeidman M, Kim PK (2016) Phytobezoars, small bowel obstruction, and intestinal infarction: The case of the grape ileus, Surgical Infections Case Reports 1:1, 8-10, DOI: 10.1089/ crsi.2016.29002.al. 